\title{
3D QSAR AND DOCKING STUDY OF INDOLE DERIVATIVES AS SELECTIVE COX-2 INHIBITORS
}

\author{
RAJASHREE CHAVAN ${ }^{*}$, HARINATH MORE ${ }^{b}$
}

aDepartment of Pharmaceutical Chemistry, Pune District Education Association's Seth Govind Raghunath Sable College of Pharmacy, Saswad, Pune 412301, India, bBharati Vidyapeeth's College of Pharmacy, Kolhapur Email: rajchavan18@gmail.com

Received: 07 Jan 2019 Revised and Accepted: 01 Mar 2019

\section{ABSTRACT}

Objective: Non-steroidal anti-inflammatory agents (NSAIDs) continue to be one of the most widely used groups of therapeutic agents. QSAR (quantitative structure-activity relationship) approach is a very useful and widespread technique for drug design. 3D QSAR facilitates evaluation of three-dimensional molecular fields around molecules and generates a relationship of these fields' values with the activity.

Methods: 3D QSAR study was performed on selected twenty-four compounds from synthesized indole derivatives using the stepwise variable selection k-nearest neighbor (kNN) molecular field analysis approach for indicating the contribution of the steric and electronic field for activity. The docking study was performed to further confirm the binding affinity of synthesized molecules (ligands) to COX-2 enzyme as well as to study binding nature.

Results: Statistically significant model was generated using VLife Molecular Design Suite 3.5 software with cross-validated correlation coefficient $\mathrm{q}^{2}$ of 0.9461 and high predictive correlation coefficient (Pred_r ${ }^{2}$ ) of 0.8782 indicating that the model is robust. The results of docking study suggest that the synthesized compounds have a comparable binding affinity with the COX-2 enzyme.

Conclusion: The present study may prove to be helpful in the development and optimization of existing indole derivatives as anti-inflammatory agents with selective COX-2 inhibition.

Keywords: Anti-inflammatory, Indole, COX-2, Docking, 3D QSAR

(C) 2019 The Authors. Published by Innovare Academic Sciences Pvt Ltd. This is an open access article under the CC BY license (http://creativecommons.org/licenses/by/4.0/) DOI: http://dx.doi.org/10.22159/ijpps.2019v11i4.31891

\section{INTRODUCTION}

Non-steroidal anti-inflammatory drugs (NSAIDs) inhibit cyclooxygenase (COX), the enzyme responsible for the conversion of arachidonic acid to prostaglandins. COX exists in 2 isoforms. COX-1 is a ubiquitous constitutive isozyme producing prostaglandins responsible for homeostatic functions such as maintenance of gastrointestinal (GI) mucosal integrity. COX-2 is largely a cytokine-induced isozyme producing prostaglandins that mediate pain and inflammation [1]. NSAIDs inhibit both COX-1 and COX-2 to varying degrees. Thus, the therapeutic effects of conventional NSAIDs are derived from inhibition of COX-2, while the adverse effects of these agents, particularly in the upper GI tract, arise from inhibition of COX-1 activity [2-3]. Much recent effort thus has been made to produce selective inhibitors of COX-2 in the belief that these will lack the gastrointestinal damaging effects of traditional NSAIDs [4-6]. Diarylheterocycle class of compounds has been investigated extensively as COX-2 inhibitors. Literature survey revealed that indole derivatives, pyrazoline derivatives, and pyrimidine derivatives independently possess good anti-inflammatory, analgesic activity and selective COX-2 inhibitory effects [7-12]. Hence we tried to obtain greater selectivity for COX-2 enzymes with the use of the indole nucleus and other structural features of different COX-2 inhibitors in the designed molecules. Thus the concept of chemical hybridization of the indole nucleus with pyrazoline and pyrimidine was attempted.

Three series of the target molecules were synthesized and subjected to preliminary pharmacological evaluation for anti-inflammatory and analgesic activity by using models like carrageenin-induced rat paw edema method and acetic acid-induced writhing in mice respectively. The compounds were also screened for acute ulcerogenicity by using Wistar rats. The compounds viz. IA7, IA9, IA11, IA12, IB3, IB7, IB12, IIA2, IIA3, IIA4, IIA5, IIA10, IIB2, IIB3, IIB4, IIB5, IIB7, IIIA4, IIIA10, IIIA11, IIIA17, IIIB10, IIIB11, IIIB17 showing comparable anti-inflammatory, analgesic activities with less ulceration were subjected to in vitro COX inhibition assays using Celecoxib as the reference [13].
The computer-aided prediction of biological activity in relation to the chemical structure of a compound is now a commonly used technique in drug discovery. Computational chemistry represents molecular structures as a numerical model and simulates their behavior with the equations of quantum and classical physics. Available programs enable scientists to easily generate and present molecular data including geometries, associated properties (electronic, spectroscopic and bulk). The usual paradigm for displaying and manipulating these data is a table in which compounds are defined by individual rows and molecular properties (or descriptors) are defined by the associated columns [14-17]

Three-dimensional quantitative structure-activity relationship (3D QSAR) facilitates evaluation of three-dimensional molecular fields around molecules and generates a relationship of these fields' values with the activity. The k-nearest neighbor ( $\mathrm{kNN}$ ) method is one of the methods for generating a relationship between activity and molecular field and provides an interpretation of results thus providing clues for designing new molecules. kNN-MFA (Molecular Field Analysis) requires suitable alignment of a set of molecules. This is followed by generation of a common rectangular grid around the molecules. The steric and electrostatic energies are computed at the lattice points of the grid using methyl probe of charge +1 . These interaction energy values at the grid points are considered for relationship generation using kNN method.

The kNN technique is a conceptually simple approach to pattern recognition problems. In this method, an unknown pattern is classified according to the majority of the class memberships of its $\mathrm{k}$ nearest neighbors in the training set. The nearness is measured by an appropriate distance metric (e. g. molecular similarity measure calculated using field interactions of molecular structures). The standard kNN method is implemented as follows: (i) calculate distances between an unknown object $(\mathrm{u})$ and all the objects in the training set (ii) select k objects from the training set most similar to object $(\mathrm{u})$, according to the calculated distances (iii) classify object (u) with the group to which a majority of the k objects belong. An 
optimal $\mathrm{k}$ value is selected by the optimization through the classification of a test set of samples or by the leave-one-out crossvalidation. The variables and optimal $\mathrm{k}$ values are chosen using different variable selection methods like Simulated Annealing variable selection method.

Drug exerts its biological activity by binding to the pocket of receptor molecule usually protein. In their binding conformations, the molecules exhibit geometric and chemical complementarily, both of which are essential for successful drug activity. The computational process of searching for a ligand that is able to fit both geometrically and energetically into the binding site of a protein is called molecular docking.

Molecular docking helps in studying drug/ligand or receptor/protein interactions by identifying the suitable active sites in the protein, obtaining the best geometry of ligand-receptor complex and calculating the energy of interaction for different ligands to design more effective ligands. The target or receptor is either experimentally known or theoretically generated through knowledge-based protein modeling or homology modeling. The molecular docking tool has been developed to obtain a preferred geometry of interaction of ligand-receptor complexes having minimum interaction energy based on different scoring functions viz. only electrostatics, the sum of steric and electrostatic and dock score. This utility allows one to screen a set of compounds for lead optimization. Genetic algorithm (GA), Piecewise Linear Pairwise Potential (PLP) and Grid algorithms are used to minimize the interaction energy between ligand-receptor.

In the present research work, a data set of twenty-four molecules showing comparable COX 2 inhibitory activity was subjected to 3D QSAR analyses, in search of newer and potent anti-inflammatory agents with selective COX-2 inhibition. Statistically, significant models were generated, and the most robust models for 3D QSAR was obtained using stepwise variable selection kNN-MFA approach using V-Life Molecular Design Suite software version 3.5.

\section{MATERIALS AND METHODS}

\section{Optimization of molecules' structures}

A data set of twenty-four molecules showing comparable COX 2 inhibitory activity measured was chosen for the present 3D QSAR study as shown in table 1 . The biological activity was expressed as $\mathrm{IC}_{50}$ values measured on COX-2 enzyme.

Table 1: The chemical structures of compounds used for 3D QSAR study<smiles>[R6]c1c(Br)c2ccccc2n1[B]</smiles>

\begin{tabular}{|c|c|c|c|c|}
\hline S. No. & Compd & $\mathbf{R}_{\mathbf{1}}$ & $\mathbf{R}_{2}$ & $\mathbf{R}_{3}$ \\
\hline 1 & IA7 & 5-(4-methoxyphenyl)-1-phenylpyrazoline & 4-chlorophenyl & $\mathrm{H}$ \\
\hline 2 & IA9 & 1,5-diphenylpyrazoline & 4-methoxyphenyl & $\mathrm{H}$ \\
\hline 3 & IA11 & 5-(4-methoxyphenyl)-1-phenylpyrazoline & 4-methoxyphenyl & $\mathrm{H}$ \\
\hline 4 & IA12 & $\begin{array}{l}\text { 5-(4-dimethyaminophenyl)-1- } \\
\text { phenylpyrazoline }\end{array}$ & 4-methoxyphenyl & $\mathrm{H}$ \\
\hline 5 & IB3 & 4(4-methoxyphenyl)pyrimidin-2-amine & Phenyl & Phenyl \\
\hline 6 & IB7 & 4(4-methoxyphenyl)pyrimidin-2-amine & 4-chlorophenyl & Phenyl \\
\hline 7 & IB12 & $\begin{array}{l}\text { 4(4-(dimethylamino) phenyl)pyrimidin-2- } \\
\text { amine }\end{array}$ & 4-methoxyphenyl & Phenyl \\
\hline 8 & IIA2 & methylsulfonyl & 5-(4-chlorophenyl)-1-phenylpyrazoline & Phenyl \\
\hline 9 & IIA3 & methylsulfonyl & (4-bromophenyl)-1-phenylpyrazoline & Phenyl \\
\hline 10 & IIA4 & methylsulfonyl & 5-(4-methoxyphenyl)-1-phenylpyrazoline & Phenyl \\
\hline 11 & IIA5 & methylsulfonyl & $\begin{array}{l}\text { 5-(4-dimethyaminophenyl)-1- } \\
\text { phenylpyrazoline }\end{array}$ & Phenyl \\
\hline 12 & IIA10 & Tosyl & $\begin{array}{l}\text { (5-(4-methoxyphenyl)-1- } \\
\text { phenylpyrazoline }\end{array}$ & Phenyl \\
\hline 13 & IIB2 & methylsulfonyl & 4-(4-chlorophenyl)pyrimidin-2-amine & Phenyl \\
\hline 14 & IIB3 & methylsulfonyl & 4-(4-bromophenyl)pyrimidin-2-amine & Phenyl \\
\hline 15 & IIB4 & methylsulfonyl & 4-(4-methoxyphenyl)pyrimidin-2-amine & Phenyl \\
\hline 16 & IIB5 & methylsulfonyl & $\begin{array}{l}\text { 4-(4-(dimethylamino)phenyl)pyrimidin- } \\
\text { 2-amine }\end{array}$ & Phenyl \\
\hline 17 & IIB7 & Tosyl & 4-phenylpyrimidin-2-amine & Phenyl \\
\hline 18 & IIIA4 & $\mathrm{H}$ & Phenyl & $\begin{array}{l}\text { 3-(4-hydroxyphenyl)-1- } \\
\text { phenylpyrazoline }\end{array}$ \\
\hline 19 & IIIA10 & $\mathrm{H}$ & 4-chlorophenyl & $\begin{array}{l}\text { 3-(4-hydroxyphenyl)-1- } \\
\text { phenylpyrazoline }\end{array}$ \\
\hline 20 & IIIA11 & $\mathrm{H}$ & 4-chlorophenyl & $\begin{array}{l}\text { 3-(4-aminophenyl)-1- } \\
\text { phenylpyrazoline }\end{array}$ \\
\hline 21 & IIIA17 & $\mathrm{H}$ & 4-methoxyphenyl & $\begin{array}{l}\text { 3-(4-aminophenyl)-1- } \\
\text { phenylpyrazoline }\end{array}$ \\
\hline 22 & IIIB10 & $\mathrm{H}$ & 4-chlorophenyl & 4-(2-amino)pyrimidin-4-yl)phenol \\
\hline 23 & IIIB11 & $\mathrm{H}$ & 4-chlorophenyl & $\begin{array}{l}\text { 4-(4-aminophenyl)pyrimidin-2- } \\
\text { amine }\end{array}$ \\
\hline 24 & IIIB17 & $\mathrm{H}$ & 4-methoxyphenyl & $\begin{array}{l}\text { 4-(4-aminophenyl)pyrimidin-2- } \\
\text { amine }\end{array}$ \\
\hline
\end{tabular}

Various approaches for the development of COX-2 inhibitors have appeared in the literature over the past several years. The methods employed include 3D QSAR studies such as comparative molecular field analysis (CoMFA), receptor surface analysis (RSA), kNN method. 
All models reported are of sufficiently high quality to be used as an estimator of the biological activity of unknown compounds; however, each model has some limitations. We attempted to obtain further insights into the structural requirement of a diarylindole class of COX2 inhibitors by the application of 3D-QSAR using kNN-MFA method. Molecular alignment was performed which is useful for studying shape variation with respect to the base structure selected for alignment. Further, the aligned molecules were used for 3D QSAR studies. The method used for alignment was Template based. A template structure was defined and used as a basis for alignment of a set of molecules. The geometries of the aligned molecules were stored in the Align Molecules subfolder in the Align folder. All the aligned molecules were opened from Align Molecules subfolder in the Align folder. The 24 aligned molecules are shown in fig. 1.

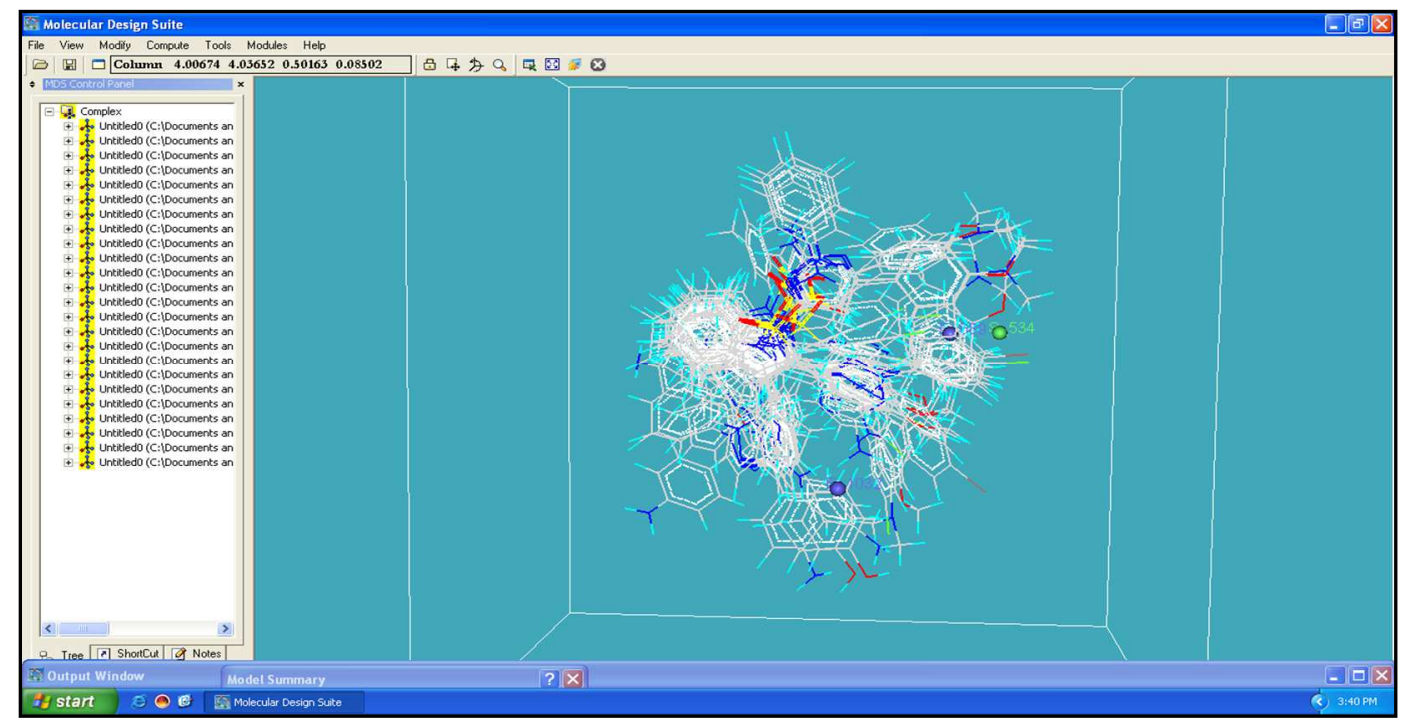

Fig. 1: Template-based alignment of 24 molecules

\section{Calculation of descriptors for 3D QSAR}

The 3D QSAR worksheet was launched at the beginning. The subfolder containing all aligned molecules was opened. The data of biological activity $\left(\mathrm{IC}_{50}\right)$ was inserted in the column. This was followed by the calculation of the molecular local shape field descriptors for finding a relationship with the activity and various parameters for field calculation selected are shown below.

\section{Field Type: Electrostatic, Steric, and Hydrophobicity}

\section{Charge Type: Gasteiger-Marsili'}

This resulted in the worksheet with 2080 field descriptors (1040 for each electrostatic and steric). For performing a robust QSAR analysis, descriptors that show variation for all the molecules are important. A descriptor that is constant for all the molecules will not contribute to QSAR and hence should be removed from the worksheet. Thus invariable columns were removed. The training and test set was selected by manual selection method ensuring that the molecules have uniform spread (training and test) in terms of both activity and chemical space. The dependent variable selected was biological activity as a negative $\log$ of $\mathrm{IC}_{50}$ and the remaining variables considered as independent variables.

The kNN-MFA model was generated using stepwise forwardbackward variable selection method. Stepwise Parameter Settings was done as follows:

Cross-Correlation Limit: 0.5

Number of Variable in Final Equation: 4

Term Selection Criteria: $\mathrm{q}^{2}$

F test In: 4

F test Out: 3.99

Variance Cut-Off: $0 \mathrm{kcal} / \mathrm{mol} \mathrm{A}^{\circ}$

\section{Scaling: Auto Scaling}

The relative positions of the local fields around aligned molecules that are important for activity variation in the model were observed by clicking the Show Points button in the 'Model Summary' dialog box. The best model was selected on the basis of various statistical parameters. The quality and predictability of the model was estimated from the cross-validated squared correlation coefficient and predicted $\mathrm{r}^{2}$.

\section{Docking study}

Molecular docking techniques consist in finding the low-energy binding modes of a ligand within the active site of a macromolecule and evaluating the binding energy with a score [18]. Explanation of the selectivity of small sets of ligands has been attempted with accurate but time-consuming techniques [19-21]. Otherwise, automated docking methods may be used to estimate the COX-2 affinity for large molecular databases. The objective of this work had been to use structural information of the target to further confirm the binding affinity of synthesized molecules to COX-2 enzyme as well as study binding nature.

\section{Optimization of protein}

Docking studies were carried out in Vlife molecular docking suite 3.5 by using Biopredicta. All the molecular modeling studies were performed on Pentium Core2Duo workstation using Sybyl. Docking studies were carried out using COX-2 (PROSTAGLANDIN SYNTHASE-2; EC: 1.14.99.1) complexed with a selective inhibitor, SC-558 (1-phenylsulphonamide-3-trifluoromethyl-5bromophenylpyrazole). The $1 \mathrm{CX} 2$ isoform of human cyclooxygenase was downloaded from Protein Data Bank website. The tetramer is converted into the monomer. Water molecules, cofactors, and heme were deleted from protein. The SC558 reference molecule was extracted. The bond order, bond angle, peptide bond check performed using the local geometry check option from the analyze menu of the BioPredicta module. Hydrogens were added in molecule and energy minimized using Merck Molecular Force Field (MMFF).

\section{Optimization of ligand}

The structures of SC558 and all the compounds used in COX-2 enzyme in vitro assay were energy minimized using MMFF until the root mean square gradient values became smaller than $0.0001 \mathrm{kcal} / \mathrm{mol} \mathrm{A}^{\circ}$. 


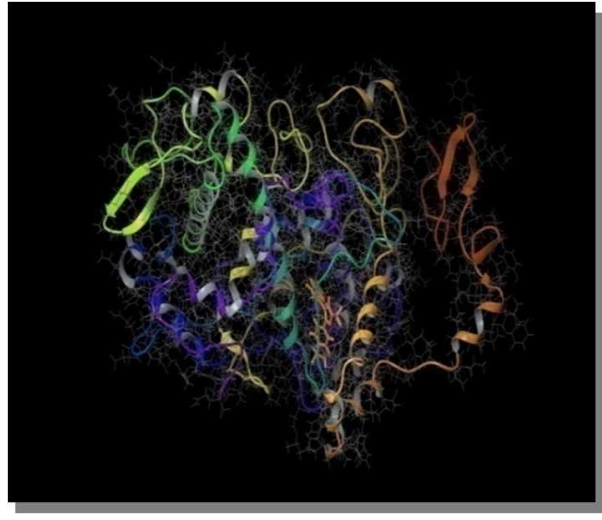

Fig. 2: 1cx2 enzyme structure

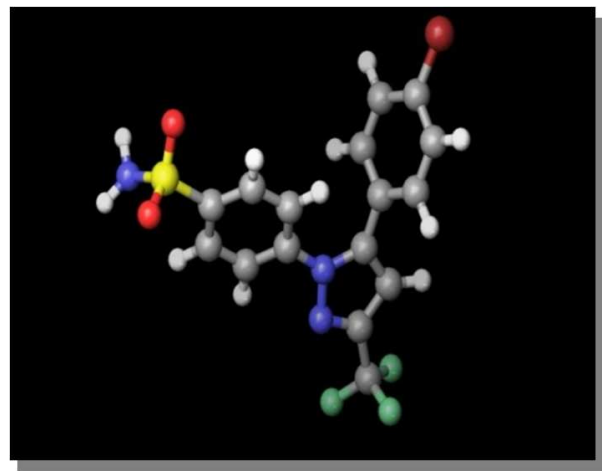

Fig. 3: Co crystalized ligand SC 558

\section{Docking}

Grid docking: This docking method can be used to dock a single ligand or its conformer with a given receptor. Cavity Number 2 in the Grid Over panel for the 1CX2 receptor selected where the docking would be performed. The default grid size was automatically chosen by VLife Molecular Design Suite. Rotation angle for ligand rotation step size set as 100. Fitness Function selected as Dock Score and No of bump allowed as 4 .
Cavity 2 in $1 \mathrm{CX} 2$ (protein and co-crystallized ligand) is the cavity where the co-crystallized ligand is located. This can be ascertained by using the Edit BioPolymer tool. Grid Docking was started. The start and progress of the grid docking process at different grid points in the cavity (docking site is chosen) was observed in the Output Window. On completion of the docking, the final minimum score (dock score interaction/docking energy of receptor-ligand) for the best ligand pose is displayed in the output window together with the location path of the docked receptor-ligand complex. Grid-based docking performed in cavity number 2 with a number of bump 4 and rotation angle $100^{\circ}$. Docking scores were recorded. After merging and energy minimization the amino acid interactions were analyzed.

\section{Structure of reference ligand SC 558}
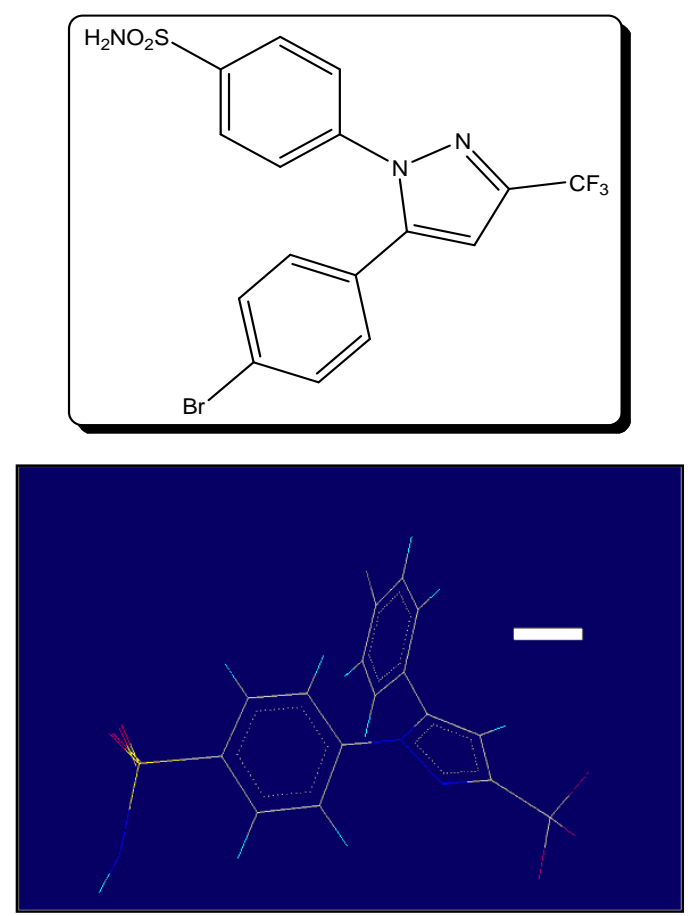

Fig. 4: Reference ligand SC 558

Table 2: In vitro COX-inhibition data of compounds

\begin{tabular}{|c|c|c|c|c|}
\hline S. No. & Compound & COX-1a (IC 50, $\mu \mathrm{M})$ & COX-2 (IC 50, $\mu \mathrm{M})$ & COX-2 SI b (Selectivity Index) \\
\hline 01 & Celecoxib & 28.6 & 0.09 & 317.77 \\
\hline 02 & IA7 & 37.8 & 2.2 & 17.18 \\
\hline 03 & IA9 & 51.2 & 3.7 & 13.83 \\
\hline 04 & IA11 & 46.8 & 3.2 & 14.62 \\
\hline 05 & IA12 & 49.6 & 2.8 & 17.71 \\
\hline 06 & IB3 & 48.1 & 3.6 & 13.36 \\
\hline 07 & IB7 & 51.5 & 3.1 & 16.61 \\
\hline 08 & IB12 & 45.6 & 2.4 & 19 \\
\hline 09 & IIA2 & 39.5 & 0.91 & 43.40 \\
\hline 10 & IIA3 & 48.6 & 0.89 & 54.60 \\
\hline 11 & IIA4 & 54.1 & 1.1 & 49.18 \\
\hline 12 & IIA5 & 47.8 & 1.3 & 36.76 \\
\hline 13 & IIA10 & 56.9 & 1.8 & 31.61 \\
\hline 14 & IIB2 & 49.9 & 0.88 & 56.70 \\
\hline 15 & IIB3 & 48.6 & 1.2 & 40.5 \\
\hline 16 & IIB4 & 53.6 & 1.02 & 52.54 \\
\hline 17 & IIB5 & 57.5 & 1.3 & 44.23 \\
\hline 18 & IIB7 & 52.8 & 1.9 & 27.78 \\
\hline 19 & IIIA10 & 44.6 & 3.1 & 14.38 \\
\hline 20 & IIIA11 & 48.1 & 3.9 & 12.33 \\
\hline 21 & IIIA17 & 50.6 & 2.6 & 19.46 \\
\hline 22 & IIIA4 & 49.1 & 3.4 & 14.44 \\
\hline 23 & IIIB10 & 39.6 & 2.3 & 17.21 \\
\hline 24 & IIIB11 & 47.6 & 1.6 & 29.75 \\
\hline 25 & IIIB17 & 38.8 & 1.1 & 35.27 \\
\hline
\end{tabular}

aValues are means of two determinations acquired using an ovine COX-1/COX-2 assay kit and the deviation from the mean is $<10 \%$ of the mean value, ${ }^{\mathrm{b}}$ In vitro $\mathrm{COX}-2$ selectivity index (COX-1 $\left.\mathrm{IC}_{50} / \mathrm{COX}-2 \mathrm{IC}_{50}\right)$. 


\section{RESULTS AND DISCUSSION}

The results of in vitro COX inhibition assay on the most active twenty-four molecules from all the series shows that these compounds are more selective towards COX-2 than COX-1 as shown in table 2. Compound IIA3, IIB2, and IIB4 from series II showed selectivity index of $54.60,56.70$ and 52.54 respectively for COX-2 vs COX-1. The compounds IIIB11 and IIIB17 from series III also showed a selectivity index of 29.75 and 35.27 respectively. The remaining compounds in series IA, IB, IIIA and IIIB have shown less selectivity index as compared to compounds in IIA and IIB series. The results show that the presence of the sulfonyl group in series IIA and IIB is favorable for maximum drug-receptor interactions. In the compounds IIIB11 and IIIB17, the presence of hydrogen bonding groups is important for optimum drug-receptor interactions.

\section{Interpretation of 3D QSAR model}

In the kNN-MFA method, several models were generated for the selected members of the training and test set and the corresponding best model is reported in table 3 .

Table 3: Statistical results of kNN molecular field analysis

\begin{tabular}{ll}
\hline Parameter & kNN-MFA method \\
\hline $\mathrm{N}$ & 16 \\
Degree of freedom & 11 \\
$\mathrm{q}^{2}$ & 0.9461 \\
$\mathrm{q}^{2}$ se & 0.0493 \\
Pred $\mathrm{r}^{2}$ & 0.8782 \\
pred_r ${ }^{2}$ se & 0.1338 \\
K/Vn & $2 / 4$ \\
Descriptors (range) & E_1436(-1.0557-0.7659) \\
& S_1207(-0.1900-0.0832) \\
& S_720(-0.7384-0.6306) \\
\hline
\end{tabular}

Results from table 3 show that predictive $\mathrm{r}^{2}$ (Pred_r ${ }^{2}$ ) for this model is 0.8782 which is considerably high and also other statistical parameters are in acceptable range.

In this model, steric and electrostatic fields' contribution was found to be 75 : $25 \%$ respectively. kNN results can be viewed as the stereo view of the molecular fields (descriptors) in a rectangular grid around the molecular unit as shown around aligned molecules.

\section{Predictivity of kNN-MFA model}

Predictivity of the model was evaluated by predicting the activity of the molecules belonging to the training set (internal predictivity) as well as molecules in the test set (external predictivity) as shown in tables 4 and 5 . Graphical representation of the predictive power of the model is shown in fig. 5.

Table 4: Observed and predicted IC 50 value data for training set compounds (16 molecules) obtained using kNN MFA method

\begin{tabular}{|c|c|c|c|c|}
\hline S. No. & Mol. ID. & Activity (Observed) & Activity (Predicted) & Residuals \\
\hline 1 & IA7 & -0.342423 & -0.363355 & 0.020932 \\
\hline 2 & IA11 & -0.505150 & -0.494736 & -0.01041 \\
\hline 3 & IB3 & -0.556303 & -0.474640 & -0.08166 \\
\hline 4 & IB7 & -0.491362 & -0.476103 & -0.01526 \\
\hline 5 & IB12 & -0.380211 & -0.337445 & -0.04277 \\
\hline 6 & IIA3 & 0.050610 & -0.029727 & 0.080337 \\
\hline 7 & IIA4 & -0.041393 & -0.228609 & 0.187216 \\
\hline 8 & IIA5 & -0.176091 & -0.221712 & 0.045621 \\
\hline 9 & IIA10 & -0.255273 & -0.249155 & -0.00612 \\
\hline 10 & IIB4 & -0.041393 & -0.249983 & 0.20859 \\
\hline 11 & IIB5 & -0.113943 & -0.060634 & -0.05331 \\
\hline 12 & IIIA4 & -0.531479 & -0.476880 & -0.0546 \\
\hline 13 & IIIA11 & -0.591065 & -0.468275 & -0.12279 \\
\hline 14 & IIIA17 & -0.414973 & -0.372382 & -0.04259 \\
\hline 15 & IIIB10 & -0.361728 & -0.352367 & -0.00936 \\
\hline 16 & IIIB11 & -0.462398 & -0.426849 & -0.03555 \\
\hline
\end{tabular}

Table 5: Observed and predicted IC $_{50}$ value data for test set compounds (8 molecules) obtained using kNN MFA method

\begin{tabular}{lllll}
\hline S. No. & Mol. ID. & Activity (Observed) & Activity (Predicted) $^{*}$ \\
\hline 1 & IA9 & -0.568202 & -0.476102 & Residuals $^{*}$ \\
2 & IA12 & -0.447158 & -0.528771 & -0.0921 \\
3 & IIA2 & 0.040959 & -0.013932 & 0.081613 \\
4 & IIB2 & 0.055517 & 0.045140 & 0.054891 \\
5 & IIB3 & -0.079181 & 0.048473 & -0.010377 \\
6 & IIB7 & -0.278754 & -0.348673 & 0.069919 \\
7 & IIIA10 & -0.491362 & -0.468257 & -0.02311 \\
8 & IIIB17 & -0.322219 & 0.358835 & 0.036616 \\
\hline
\end{tabular}




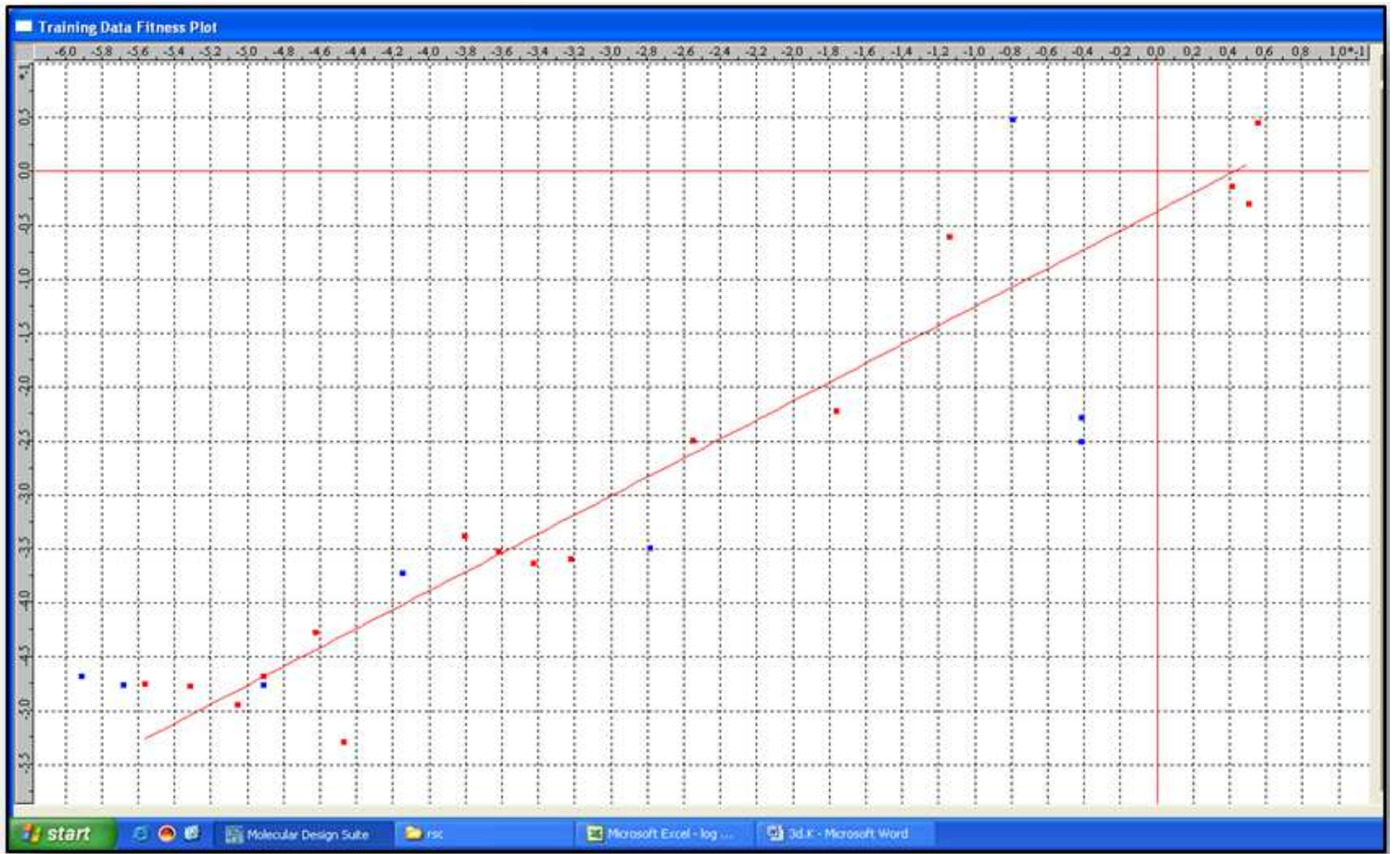

Fig. 5: Plot of observed vs. predicted activity $\left(\mathrm{PIC}_{50}\right)$ ) of the molecules in the training and test set by the stepwise kNN-MFA method

\section{Discussion of stereo view plot of kNN MFA method}

Stereo view of the molecular fields (descriptors) in a rectangular grid around the aligned molecules is shown in fig. 6.

The green spheres show the location where steric contribution has played an important role and variation in bulk in that region would help in improving the activity. Similarly, blue spheres are the locations where electrostatic contribution has played an important role and the variation in electronegativity in that region would help in improving the activity. The first value in the parenthesis indicates the lower limit and the second value is the upper limit of steric/electrostatic function values for molecules. The molecule adhering to these ranges at respective field locations are likely to be in the higher activity range.

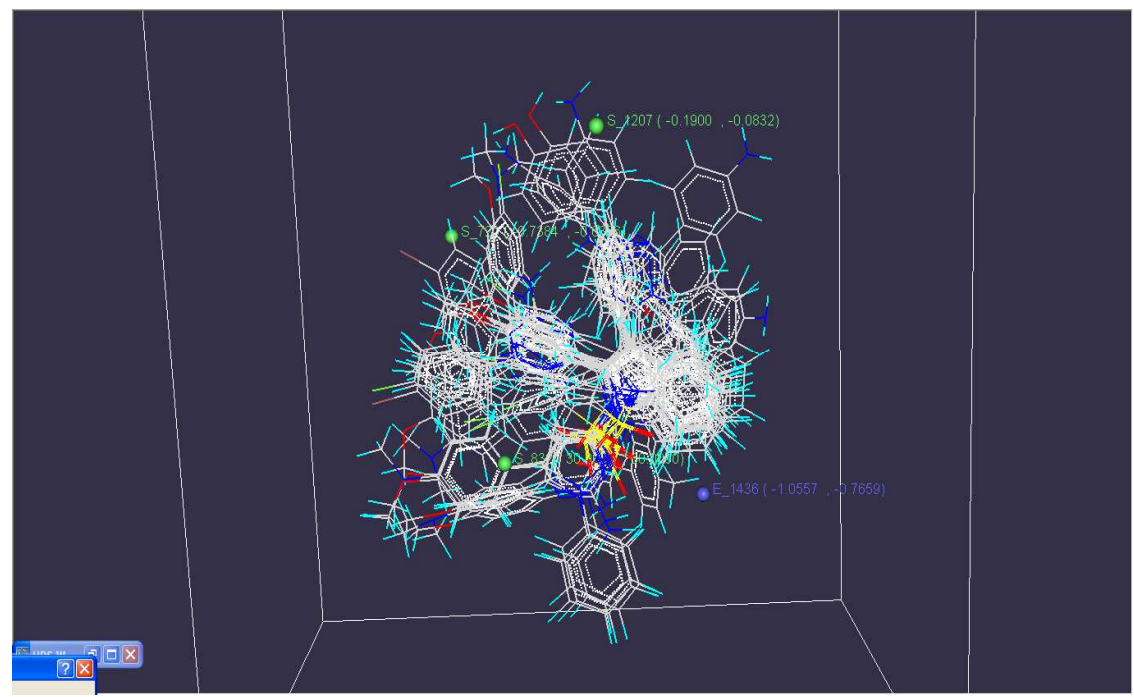

Fig. 6: Stereoview of the molecular fields (Descriptors) in a rectangular grid around aligned molecules using kNN MFA method

1) Electrostatic field:

a) Negative range indicates that negative electrostatic potential is favorable for an increase in the activity and hence a more electronegative substituent group is preferred in the region. b) Positive range indicates that positive electrostatic potential is favorable for an increase in the activity and hence a less electronegative substituent group is preferred in that region.

2) Steric field: 
a) Negative range indicates that negative steric potential is favorable for an increase in the activity and hence a less bulky substituent group is preferred in the region.

b) Positive range indicates that positive steric potential is favorable for an increase in the activity and hence a more bulky substituent group is preferred in that region.

A green sphere (S 832) near the sulfonamide moiety and aryl group at 1 position of indole ring, suggests a more bulky group is required in this region as indicated by more positive values in the paranthesis. Another green sphere (S 720) at the para position of aryl ring at 2-position of indole ring, suggests less bulky groups are favored for activity in this region. Green sphere (S 1207) at the para position on aryl ring at 3-position of indole ring suggests that addition of bulk here may decrease the activity as a value in the parentheses is more towards the negative side.

Only one blue sphere (E 1436) is observed in the model near the para position of aryl ring at position 1 of the indole nucleus. Negative values in the parentheses suggest the more electronegative group is favorable in this position.

Molecules were optimized using MMFF method till gradient convergence $0.05 \mathrm{kcal} / \mathrm{mol}$. For viewing the results of docking, compound IIIB17 has been selected according to the best-scored conformation predicted by the scoring function. The interaction of this ligand has been shown in fig. 7. The reference ligand SC558 docked in cavity 2 of a 1CX2 isoform of the cyclooxygenase enzyme is shown in fig. 8.

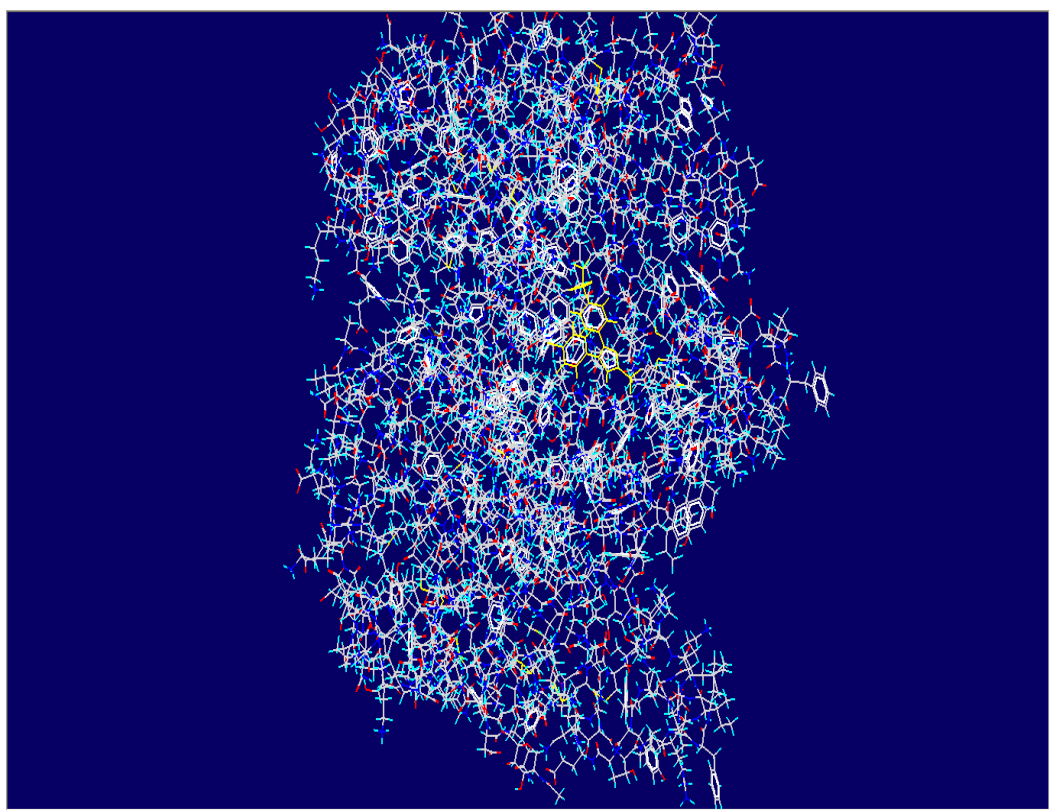

Fig. 7: Docking of reference compound in cavity 2 of the 1CX2 isoform of Cyclooxygenase enzyme

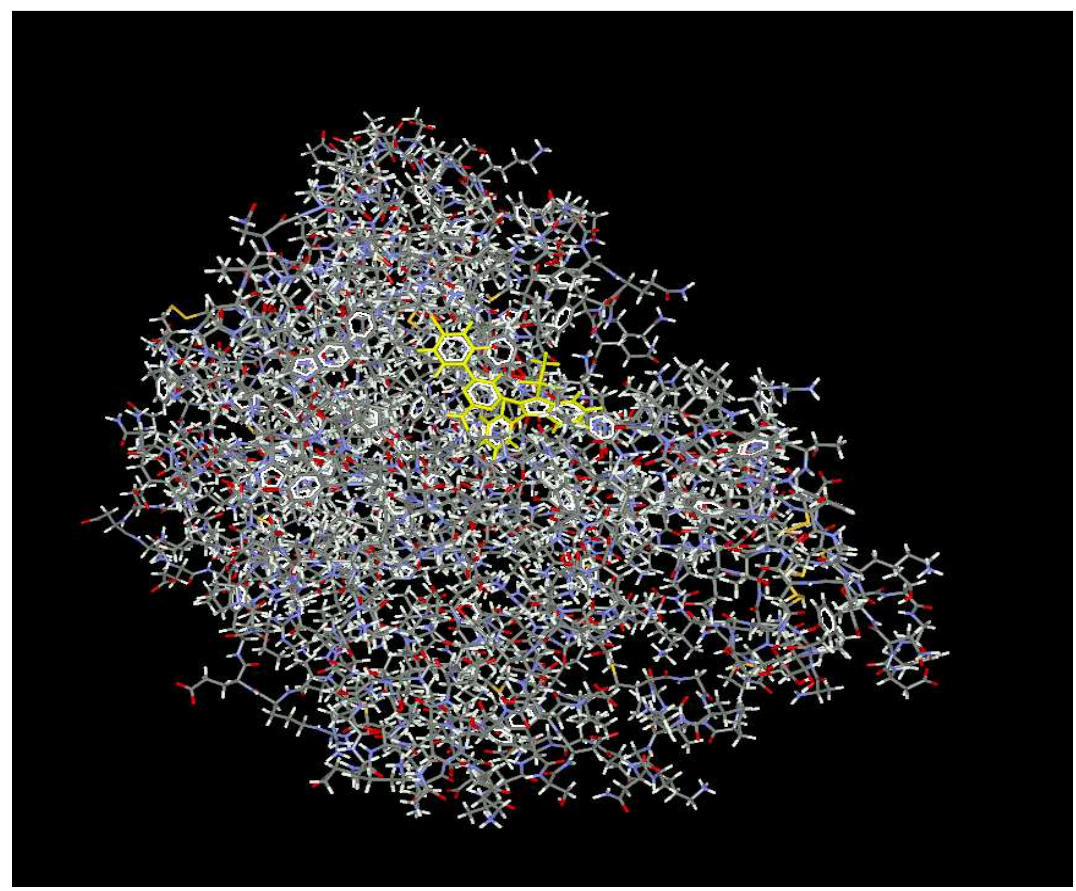

Fig. 8: Docking of the most active compound (IIIB17) in cavity 2 of the 1CX2 isoform of Cyclooxygenase enzyme 


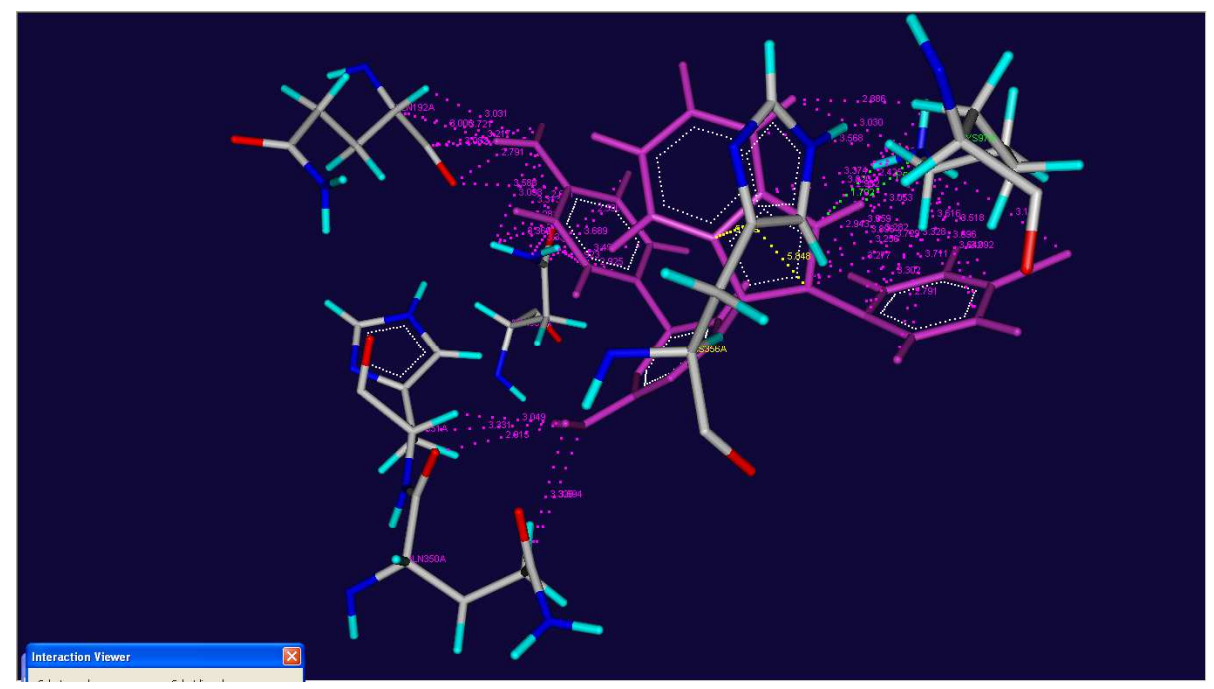

Fig. 9: Binding of IIIB17 into the active site of the COX-2 enzyme

The results have been discussed mainly with respect to IIIB17 which have better selectivity towards COX-2 enzyme. Hydrogen bonding is shown as yellow dotted lines and good contacts as dotted pink lines. Good contacts are viewed in $5 \mathrm{~A}^{\circ}$ area. The best docking pose observed for compound IIIB17 was IIIB17_P16 and docking score was-14.53 which is comparable to the docking score of original ligand-14.98. The lower interaction energy observed for IIIB17 rationalizes the tighter binding of indole analog. The compound IIIB17 was found to be involved in the hydrogen bonding with a residue Lys 97.

Table 6: Docking scores of compounds with 1cx2_series A mol 2 receptor

\begin{tabular}{|c|c|c|c|}
\hline S. No. & Compound & Docking score & Binding energy \\
\hline 1 & SC558 & -14.98 & -46.2 \\
\hline 2 & IIIB17 & -14.53 & -21.3 \\
\hline 3 & IIB2 & -14.42 & -27.4 \\
\hline 4 & IIIB11 & -14.3 & -12.4 \\
\hline 5 & IIA2 & -13.81 & -51.5 \\
\hline 6 & IIB4 & -12.7 & -24.2 \\
\hline 7 & IIA4 & -12.43 & -13.9 \\
\hline 8 & IIB3 & -12.4 & -16.4 \\
\hline 9 & IIA5 & -11.33 & -24.9 \\
\hline 10 & IIB5 & -11.12 & -39.4 \\
\hline 11 & IIA10 & -11.12 & -10.9 \\
\hline 12 & IIB7 & -11.11 & -16.6 \\
\hline 13 & IIA3 & -10.02 & -22.9 \\
\hline 14 & IA7 & -10 & -17.5 \\
\hline 15 & IIIB10 & -10.98 & -11.5 \\
\hline 16 & IB12 & -9.88 & -12.3 \\
\hline 17 & IIIA17 & -9.88 & -5.6 \\
\hline 18 & IA12 & -9.76 & -4.4 \\
\hline 19 & IB7 & -8.61 & -8.3 \\
\hline 20 & IIIA10 & -8.59 & -15.5 \\
\hline 21 & IA11 & -7.47 & -4 \\
\hline 22 & IIIA4 & -7.32 & -13.4 \\
\hline 23 & IB3 & -7.26 & -14 \\
\hline 24 & IA9 & -7.06 & -19 \\
\hline 25 & IIIA11 & -6.04 & -1.3 \\
\hline
\end{tabular}

The hydrogen bonding interaction is as follows

\begin{tabular}{llll}
\hline S. No. & Residue atom & Ligand atom & Distance \\
\hline 1 & LYS97A 514N & $9059 \mathrm{H}$ & $1.599 \mathrm{~A}^{\circ}$ \\
2 & LYS97A 5044H & $9031 \mathrm{~N}$ & $1.792 \mathrm{~A}^{\circ}$ \\
\hline
\end{tabular}

The hydrogen bonding distance between NH group of IIIB17 with $\mathrm{NH}$ of Lysine 97A was found to be $1.599 \mathrm{~A}^{\circ}(\mathrm{N}----\mathrm{H})$.

The pyrimidine ring at 3 positions was surrounded by active site amino acid residues His 356A, Gly 350A, His 351A, and Asn 581A. The indole ring found to interact with residues His 356A, Lysine97A.
The benzene ring on the pyrimidine nucleus was found to interact with Asn581A and Gly 192A.

Twenty four molecules from six series viz IA, IB, IIA, IIB, IIIA, and IIIB were studied by docking with an aim to reveal their binding interactions with COX-2 enzyme. Compounds IIIB17, IIIB11 and IIB2 
shown good docking score comparable to SC558, confirms the results obtained through COX inhibition assays.

\section{CONCLUSION}

The present 3D QSAR study using kNN-MFA method indicated the contribution of steric and electronic fields in the activity of compounds. The model suggests that a more bulky group is required near the sulfonamide moiety/aryl group at 1 position of indole ring, as indicated by positive values in the parenthesis and less bulky groups are favored for activity at the para position of aryl ring at 2position of indole ring while addition of bulk at the para position on aryl ring at 3-position of indole ring may decrease the activity. The model further suggests that the more electronegative group is favorable at the para position of aryl ring at position 1 of the indole nucleus. The results of docking studies indicate that the compound IIIB17 with highest docking score was found to be involved in the hydrogen bonding with a residue Lys 97 . The hydrogen bonding distance between $\mathrm{NH}$ group of IIIB17 with $\mathrm{NH}$ of lysine97A was found to be $1.599 \mathrm{~A}{ }^{\circ}(\mathrm{N}-----\mathrm{H})$. Thus the results obtained can be used for further modification and optimization of the indole derivatives for better anti-inflammatory activity with selective COX2 inhibition.

\section{ACKNOWLEDGMENT}

The authors are grateful to Principal Dr. Ashok V. Bhosale for providing infrastructure and facilities to conduct the research activity. We are also thankful to VLife Sciences for providing technical support from time to time.

\section{AUTHORS CONTRIBUTIONS}

All the authors have contributed equally

\section{CONFLICTS OF INTERESTS}

All authors have none to declare

\section{REFERENCES}

1. Gierse JK, McDonald JJ, Scott D, Shaukat H, Koboldt CM, Seibert KA. Single amino acid difference between cyclooxygenase-1 (COX-1) and-2 (COX-2) reverses the selectivity of COX-2 specific inhibitors. J Biol Chem 1996;271:15810-4.

2. Maheshwari PPD, Ravichandiran V. Adverse drug reactions and interactions of NSAIDs in general care hospital. Asian J Pharma Clin Res 2014;7:69-71.

3. Marnett LJ, Kalgutkar AS. Cyclooxygenase 2 inhibitors: discovery selectivity and the future. Trends Pharmacol Sci 1999;20:465-9.

4. Ross E. Goodman and gilman's the pharmacological basis of therapeutics. $10^{\text {th }}$ Edition. McGraw-Hill Medical Publishing Division; New York; 2000. p. 31-8.

5. Mahajan A, Sharma R. COX-2 selective nonsteroidal antiinflammatory drugs: current status. J Assoc Physicians India 2005;53:2001-4.

6. Saxena AK, Ramachandran R, Gulati M, Kumar M. Cyclooxygenase-2 inhibitors in postoperative pain and chronic pain management: current opinion and future perspective. Indian J Anaesth 2005;49:170-9.

7. Amir M, Dhar N, Tiwari S. Synthesis and anti-inflammatory, analgesic, ulcerogenic and lipid peroxidation activities of 3,5dimethyl pyrazoles,3-pyrazole-5-ones, and 3,5-disubstituted pyrazolines. Indian J Chem Sect B 1997;36B:2532-7.

8. Barsoum FF, Girgis AS. Facile synthesis of bis (4,5-dihydro1Hpyrazole-1-carboxamides) and their thio-analogs of potential PGE2 inhibitory properties. Eur J Med Chem 2009;44:2172-7.

9. Cenicola ML, Donnoli D, Stella L, Paola CD, Constantino M, Anignente $\mathrm{E}$, et al. Research on heterocyclic compounds. Antiinflammatory activity of some imidazo(1,2-c)pyrimidine derivatives. Pharmacol Res 1990;22:80-4.

10. Nargund LVG, Badiger VV, Yarnal SM. Synthesis and antimicrobial and anti-inflammatory activities of substituted 2mercapto-3-( $n$-aryl)pyrimido[5,4-c]cinnolin-4-(3H)-ones. J Pharm Sci 1992;81:365-6.

11. Kalgutkar AS, Marnett AB, Crews BC, Remmel RP, Marnett LJ. Ester and amide derivatives of the nonsteroidal antiinflammatory drug, indomethacin, as selective COX-2 inhibitors. J Med Chem 2000;43:2860-70.

12. Rani P, Srivastava VK, Kumar A. Synthesis and antiinflammatory activity of heterocyclic indole derivatives. Eur J Med Chem 2004:39:449-52.

13. Husain A, Ahmad A, Alam MM, Ajmal Mohd, Ahuja P. Fenbufen based 3-[5-(substituted aryl)-1,3,4-oxadiazol-2-yl]-1(biphenyl-4-yl)propan-1-ones as safer anti-inflammatory and analgesic agents. Eur J Med Chem 2009;44:3798-804.

14. Katritzky A. QSAR modeling, synthesis, and bioassay of diverse leukemia RPMI-8226 cell line active agents. Bioorg Med Chem Lett 2010;45:5183-99.

15. Rapatri V, Chitre T, Bothara K. Novel 4-(morpholin-4-yl)-N(arylidene) benzohydrazides: synthesis, antimycobacterial activity, and QSAR investigations. Eur J Med Chem 2009;44:3954-60.

16. Karthiga Devi S, Velmurugan D. Molecular modelling, QSAR and pharmacophore studies on anti-viral, anti-malarial and antiinflammatory bioactive compounds from marine sources. Asian J Pharma Clin Res 2015;8:36-43.

17. VLife Molecular Design Suite version 3.5; VLife Sciences Technologies Pvt. Ltd., Pune, India; 2010.

18. Habeeb AG, Parveen PNR, Knaus EE. Design and synthesis of 4, 5-diphenyl-4-isoxazolines: novel inhibitors of cylooxygenase-2 with analgesic and anti-inflammatory activity. J Med Chem 2001;44:2921-7.

19. Palomer A, Perez JJ, Navea S, Llorens O, Pascual J, Garcia L, et al. Modeling cyclooxygenase inhibition: implication of active site hydration on the selectivity of ketoprofen analogues. J Med Chem 2000;43:2280-4.

20. Price MLP, Jorgensen WL. Analysis of binding affinities for celecoxib analogs with COX-1 and COX-2 from combined docking and monte carlo simulations and insight into the COX2/COX-1 selectivity. J Am Chem Soc 2000;122:9455-66.

21. Price MLP, Jorgensen WL. The rationale for the observed COX2/COX-1 selectivity of celecoxib from monte carlo simulation. Bioorg Med Chem Lett 2001;11:1541-4. 